its arguments more alive. It is also a pity that so little attention is given to children in the report: a pity because, in the realm of mental health, prevention or earlier intervention is so much better than later intervention. The statement on p I that 'far too little is yet known about the causes of [troublesome] behaviour' is misleading. Of course we must modestly acknowledge our ignorance, but we do know enough to know that there are a number of measures that society could take to improve the care of babies and young children, and to reduce further the number of unwanted children, thereby reducing the incidence of serious personality disorder and mental illness later in life, and preventing at least some of the ethical headaches that consequently arise. But that is something for another working party.

IAIN DRESSER

Department of Child Psychiatry Paddington Green Children's Hospital

London

\section{Death and Dying, a Quality of Life}

\author{
Eds Patricia F Pegg and Erno Metze \\ Pitman, London \\ £I 5
}

This book is made up from the papers presented at the International Congress on Death and Dying held in London in 1980. The proceedings of such gatherings are often disjointed and indigestible, but here is the exception which proves the rule. Having said that, it is easy to take issue with the order in which the papers are presented. One can sympathise with the editor's dilemma: is it best to proceed from the general to the particular and risk the reader losing interest in what appears to be the main thrust of the book or to start with the particular and risk not seeing the wood for the trees?

As the structure stands the first half of the book deals almost solely with bereavement and the reader began to wonder what the sub-title meant. It is not that the earlier papers are not interesting, but somehow they engendered a feeling of impatience because they seemed to be setting the scene at a pace reminiscent of the opening of a novel by Sir Walter Scott. No doubt on second reading the early chapters would come into their own; they are just in a different key from the middle movement of papers which are narrower in scope but have a freshness and immediacy which will quickly engage the attention of professionals working in the field. The final movement returns to bereavement but in more specific contexts which are of great interest and value.

Not that the early papers are unimportant or uninteresting. The primitive societies' rituals to placate the ghosts of the dead are seen to be equivalent to the painful disengagement of the bereaved member of a Western culture from the emotional bonds with the deceased. This not only illuminates what is happening for an interested professional, but also opens the way to understanding how communities respond to bereavement, which is described in the closing chapters.

Inevitably there is overlap, but this is not irritating. Manocchio's chapter covers most of the field, but the fact that there is a section on the stillborn child only serves to emphasise the importance of this problem (dealt with in two other papers) which, until recently, has been almost totally neglected by the professionals concerned. As in many other areas, only the personal experience of a professional of having a stillborn child may stimulate an enquiry into why stillbirths have been swept under the carpet. The same might also be said of cot-deaths. This section is particularly valuable for professionals who may only come across such disasters occasionally, and who therefore may feel at sea with the emotional problems of the parents. Sudden death in adults is much commoner and some experience may be available, but the chapters on sudden death and disasters are valuable for their analysis of the needs of the bereaved.

The chapters on coping with the fears of recurrence and death, together with Metze's chapter on the coronary patient living with the possibility of sudden death, are very exciting. They attempt to break new ground while at the same time remaining extremely practical. The concept of the need for the patient who has developed cancer recurrence to put his mind inte 'neutral' is fascinating and opens up an alternative to the paths of denial or defeat. The chapter is strengthened by the case histories.

These chapters are set off by the chapter by Schneider which seeks to conceptualise the process of bereavement and provides interesting and useful maps of the country through which the journey has to be undertaken.
This brief survey must inevitably seem to be invidious to those authors whose papers have not been touched on - notably absent are those from Antonius-Ijsselmonde - but this is only because the hospice concept is perhaps more firmly established in the UK and therefore appears less striking, although the Dutch workers are breaking new ground.

Altogether it is remarkable that a collection of authors working in different countries and on different lines can produce a satisfying feeling of synthesis in the mind of the reader. Perhaps it is a pity that the title and sub-title were not reversed!

\section{MICHAEL COURTENAY Bridge Lane Health Centre, London SW11}

\section{Bereavement Visiting}

\section{Edited by G Dyne}

King's Fund Publishing Office, London

$$
£ 2.85
$$

This attractively produced small book describes the bereavement visiting service at St Christopher's Hospice, London. It is not a textbook about grief, though the interested reader is given a number of suggestions about further reading. The service started in $197 \mathrm{I}$, initially in a small way. Visitors come from varied backgrounds but all have worked at the hospice in some capacity, either as a volunteer or on the staff. Volunteers make good visitors but, as with staff members, there is need for support. This is given mainly by a monthly review meeting. Access to a social worker and psychiatrist is also available should this be necessary.

Advice is given about arranging a visit and the reader is given an indication of what might happen during one. Three case studies add much to the value of the book. The final section describes the Pilgrim Club, an informal weekly meeting for the bereaved. All in all this is a helpful book for those working in similar settings. The need to care for the bereaved will always be with us.

ROBERT G TWYCROSS

Sir Michael Sobell House The Churchill Hospital Headington 